AperTO - Archivio Istituzionale Open Access dell'Università di Torino

\title{
Exceptionally Low Shear Modulus in a Prototypical Imidazole-Based Metal-Organic Framework
}

\section{This is the author's manuscript}

Original Citation:

Availability:

This version is available http://hdl.handle.net/2318/101802

since 2016-08-04T13:25:21Z

Published version:

DOI:10.1103/PhysRevLett.108.095502

Terms of use:

Open Access

Anyone can freely access the full text of works made available as "Open Access". Works made available under a Creative Commons license can be used according to the terms and conditions of said license. Use of all other works requires consent of the right holder (author or publisher) if not exempted from copyright protection by the applicable law. 


\title{
Exceptionally Low Shear Modulus in a Prototypical Imidazole-Based Metal-Organic Framework
}

\author{
Jin-Chong Tan, ${ }^{1}$ Bartolomeo Civalleri, ${ }^{2}$ Chung-Cherng Lin, ${ }^{3}$ Loredana Valenzano, ${ }^{2,4}$ Raimondas Galvelis, ${ }^{5}$ Po-Fei Chen, ${ }^{6}$ \\ Thomas D. Bennett, ${ }^{1}$ Caroline Mellot-Draznieks, ${ }^{5,7}$ Claudio M. Zicovich-Wilson, ${ }^{8}$ and Anthony K. Cheetham ${ }^{1, *}$ \\ ${ }^{1}$ Department of Materials Science and Metallurgy, University of Cambridge, Pembroke Street, \\ Cambridge CB2 3QZ, United Kingdom \\ ${ }^{2}$ Department of Inorganic, Physical, and Materials Chemistry, NIS Centre of Excellence, \\ and INSTM Reference Center, University of Turin, via Pietro Giuria 7, 10125 Torino, Italy \\ ${ }^{3}$ Institute of Earth Sciences, Academia Sinica, Nankang, Taipei, 115 Taiwan \\ ${ }^{4}$ Department of Physics, Michigan Technological University, 1400 Townsend Drive, Houghton, Michigan 49931-1295, USA \\ ${ }^{5}$ Department of Chemistry, University College London, 20 Gordon Street, London, WCH1 OAJ, United Kingdom \\ ${ }^{6}$ Department of Earth Sciences and Graduate Institute of Geophysics, National Central University, Jhongli, 32001 Taiwan \\ ${ }^{7}$ Laboratoire de Chimie et Biologie des Métaux, Université Joseph Fourier, Grenoble, CNRS, UMR 5249, CEA, DSV/iRTSV, \\ 17 rue des Martyrs, F-38054, Grenoble cedex 9, France \\ ${ }^{8}$ Facultad de Ciencias, Universidad Autonoma del Estado de Morelos, Avenida Universidad 1001, 62209 Cuernavaca, Mexico
} (Received 14 December 2011; published 29 February 2012)

\begin{abstract}
Using Brillouin scattering, we measured the single-crystal elastic constants $\left(C_{i j}\right.$ 's $)$ of a prototypical metal-organic framework (MOF): zeolitic imidazolate framework (ZIF)-8 [Zn(2-methylimidazolate) $\left.{ }_{2}\right]$, which adopts a zeolitic sodalite topology and exhibits large porosity. Its $C_{i j}$ 's under ambient conditions are (in GPa) $C_{11}=9.522(7), C_{12}=6.865(14)$, and $C_{44}=0.967(4)$. Tensorial analysis of the $C_{i j}$ 's reveals the complete picture of the anisotropic elasticity in cubic ZIF-8. We show that ZIF-8 has a remarkably low shear modulus $G_{\min } \lesssim 1 \mathrm{GPa}$, which is the lowest yet reported for a single-crystalline extended solid. Using $a b$ initio calculations, we demonstrate that ZIF-8's $C_{i j}$ 's can be reliably predicted, and its elastic deformation mechanism is linked to the pliant $\mathrm{ZnN}_{4}$ tetrahedra. Our results shed new light on the role of elastic constants in establishing the structural stability of MOF materials and thus their suitability for practical applications.
\end{abstract}

DOI: 10.1103/PhysRevLett.108.095502

Metal-organic frameworks (MOFs) [1] are crystalline microporous materials noted for their enormous structural diversity and chemical versatility. Given their vast potential for gas storage, separations, sensing, and drug delivery applications [2], detailed knowledge of the mechanical behavior of MOFs is highly desirable. There is, however, a major lack of understanding about their fundamental mechanical characteristics [3]. For example, experimental studies on the elastic constants $\left(C_{i j}\right.$ 's) of MOFs have not been documented and, so far, there are no attempts to uncover other essential elastic properties like the shear modulus $(G)$ and Poisson's ratio $(\nu)$. Tensorial analysis of the $C_{i j}$ 's allows not only the full characterization of the anisotropic nature of elasticity in MOFs but also the identification of any anomalous elastic behavior (e.g., negative Poisson's ratio [4]). Indeed, the $C_{i j}$ 's are fundamentally important, as they reflect the intrinsic interatomic bonding that governs the structure and stability of solids. Although theoretical predictions of the $C_{i j}$ 's of several MOFs have emerged (e.g., MOF-5 [5,6]), their accuracy has not been experimentally verified.

Zeolitic imidazolate frameworks (ZIFs) $[7,8]$ represent a major subfamily of MOFs that is highly topical. ZIFs combine the thermal and chemical stability of inorganic zeolites with the rich topological diversity and pore size
PACS numbers: 62.20.D-, 63.20.dk, 78.35.+c, 81.07.Pr

tunability characteristic of MOFs. The structures of ZIFs comprise tetrahedral metal centers $\left(M^{n+}=\mathrm{Zn}^{2+}, \mathrm{Co}^{2+}\right.$, $\mathrm{Li}^{+}, \mathrm{B}^{3+}$ ) bridged by imidazolate-derived linkers (e.g., $\left.\operatorname{Im}=\mathrm{C}_{3} \mathrm{~N}_{2} \mathrm{H}_{3}^{-}\right)$to form $M(\operatorname{Im})_{n}$ open frameworks adopting zeolitic architectures. Particularly, the $M$-Im- $M$ bridging linkages in ZIFs subtend an angle of $\sim 145^{\circ}$ at the Im ring center, analogous to the $\mathrm{Si}-\mathrm{O}-\mathrm{Al}$ angle in conventional aluminosilicate zeolites [9]. In this Letter, we elucidate the complete elasticity profile of a prototypical ZIF with the sodalite (SOD) topology, ZIF-8 $\left[\mathrm{Zn}(\mathrm{mIm})_{2} ; \mathrm{mIm}=\right.$ 2-methylimidazolate] [8], through experimental and theoretical approaches. ZIF-8 crystallizes in the cubic space group $I \overline{4} 3 m$ (lattice constant $16.992 \AA$ ) and exhibits a large pore volume exceeding $2400 \AA^{3}$ per unit cell (solvent accessible volume, SAV 50\% [10]).

We measured the single-crystal elastic constants $\left(C_{i j}\right.$ 's) of ZIF-8 using Brillouin scattering. Desolvated single crystals, free from twinning and cracks, were used to prepare two optically clear specimens that intersect all three crystallographic axes [9]. Brillouin spectra of ZIF-8 under ambient conditions are shown in Figs. 1(a) and 1(b). For each specimen, a total of at least 90 longitudinal $(L)$ and transverse $(T)$ acoustic wave velocities over 19 unique crystallographic orientations were used to characterize the velocity surfaces [Figs. 1(c) and 1(d)]. The most striking feature of the spectra is that both slow and fast transverse 

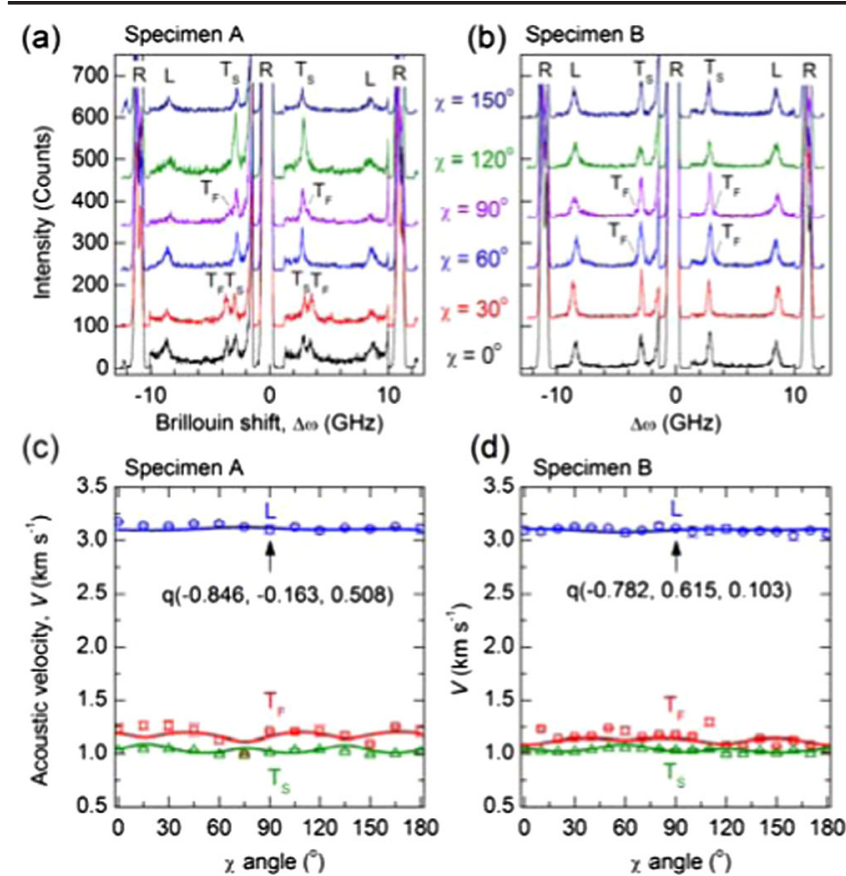

(d)

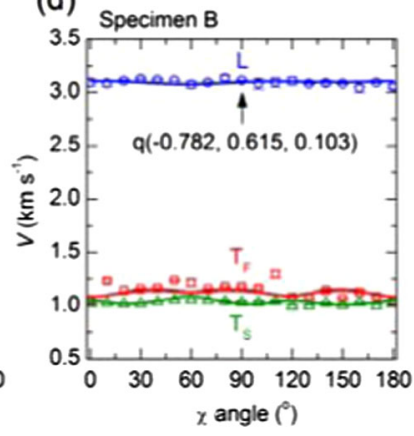

FIG. 1 (color online). (a),(b) Representative Brillouin spectra as a function of frequency shift over $\chi$ of $180^{\circ}$, for specimens $A$ and $B$, respectively. " $R$ " is the elastically scattered central Rayleigh peak; the signals around $\pm 2 \mathrm{GHz}$ are unattenuated tails of the Rayleigh peaks. (c),(d) The symbols with error bars denote the measured acoustic velocities. The curves are calculated from the best-fit $C_{i j}$ 's. " $q$ " is the phonon direction at $\chi=90^{\circ}$ (see [9]). acoustic modes $\left(T_{\mathrm{S}}\right.$ and $\left.T_{\mathrm{F}}\right)$ travel at extremely low velocities of just 1.0 to $1.2 \mathrm{~km} \mathrm{~s}^{-1}$. Likewise, the maximum velocity of the longitudinal waves $(L)$ remains below $3.2 \mathrm{~km} \mathrm{~s}^{-1}$. Such low acoustic velocities $(V)$ reflect low elastic stiffnesses $(C)$ of the underlying structure, for which the latter is the product of mass density $(\rho)$ and the square of velocity, i.e., $C=\rho V^{2}$ [11]. Following inversion of the acoustic velocity data [9], we established the three independent elastic constants $C_{11}, C_{12}$, and $C_{44}$ (Table I), which fully describe the elasticity of ZIF-8.

For comparison with the experimental results, we used $a b$ initio quantum mechanical computations (CRYSTAL09 code $[9,12])$ to calculate ZIF-8's $C_{i j}$ 's. These, and the associated elastic properties derived from the $C_{i j}$ 's, are presented in Tables I and II. The theoretical results highlight that ab initio calculations based on the B3LYP hybrid functional [13] provide reliable estimations over a wide range of mechanical properties. We note, however, an overestimation in the $C_{11}$ and $C_{12}$ stiffness coefficients (15-20\%), which in turn yield a higher bulk modulus $K(\sim 19 \%)$. This result can be attributed to the neglect of thermal effects and zero-point motion $[3,11]$ in the present theoretical treatment $(0 \mathrm{~K})$. Importantly, both experiments and theory confirm that the $C_{i j}$ 's of ZIF-8 are such that they satisfy the fundamental elastic stability criteria, for which the restrictions imposed on a cubic crystal are [14] $C_{11}>\left|C_{12}\right|, C_{11}+2 C_{12}>0$, and $C_{44}>0$.

TABLE I. Single-crystal elastic properties of ZIF-8 measured by Brillouin scattering compared with theoretical predictions (perfect crystal). The elastic compliance coefficients $S_{i j}$ were determined through inversion of the $C_{i j}$ 's; the very large magnitude of $S_{44}$ signifies an exceedingly low resistance against shear deformation. Additionally, single-crystal nanoindentation experiments were performed to measure the "indentation moduli"; see [9] for details.

\begin{tabular}{|c|c|c|c|c|}
\hline \multirow[b]{2}{*}{ Elastic properties } & & \multicolumn{2}{|c|}{ Experimental data $(295 \mathrm{~K})$} & \multirow{2}{*}{$\begin{array}{c}A b \text { initio calculations } \\
\text { B3LYP }(0 \mathrm{~K})\end{array}$} \\
\hline & & Brillouin scattering & Nanoindentation & \\
\hline \multirow{3}{*}{$\begin{array}{l}\text { Stiffness coefficient, } \\
C_{i j}(\mathrm{GPa})\end{array}$} & $C_{11}$ & $9.5226 \pm 0.0066$ & \multirow{3}{*}{$\cdots$} & 11.038 \\
\hline & $C_{12}$ & $6.8649 \pm 0.0144$ & & 8.325 \\
\hline & $C_{44}$ & $0.9667 \pm 0.0044$ & & 0.943 \\
\hline \multirow{3}{*}{$\begin{array}{l}\text { Compliance } \\
\text { coefficient, } S_{i j}\left(\mathrm{GPa}^{-1}\right)\end{array}$} & $S_{11}$ & 0.2652 & \multirow{3}{*}{$\cdots$} & 0.2578 \\
\hline & $S_{12}$ & -0.1111 & & -0.1108 \\
\hline & $S_{44}$ & 1.0345 & & 1.0605 \\
\hline \multirow{2}{*}{$\begin{array}{l}\text { Acoustic wave } \\
\text { velocities, } V\left(\mathrm{~km} \mathrm{~s}^{-1}\right)\end{array}$} & $\begin{array}{c}\text { Longitudinal } \\
\text { (maximum and minimum) }\end{array}$ & $3.17 \& 3.08$ & \multirow{2}{*}{$\cdots$} & $3.41 \& 3.32$ \\
\hline & $\begin{array}{c}\text { Transverse } \\
\text { (maximum and minimum) }\end{array}$ & $1.18 \& 1.01$ & & $1.19 \& 1.00$ \\
\hline \multirow{3}{*}{ Young's modulus, E (GPa) } & $E_{\max }=E\{100\}$ & $3.77 \pm 0.01$ & $3.29 \pm 0.11$ & 3.879 \\
\hline & $E\{110\}$ & $2.98 \pm 0.01$ & $3.07 \pm 0.07$ & 2.953 \\
\hline & $E_{\min }=E\{111\}$ & $2.78 \pm 0.01$ & $2.87 \pm 0.09$ & 2.736 \\
\hline \multirow{2}{*}{ Shear modulus, $G(\mathrm{GPa})$} & $G_{\max }=1 / 2\left(C_{11}-C_{12}\right)$ & $1.329 \pm 0.005$ & \multirow{2}{*}{$\cdots$} & 1.36 \\
\hline & $G_{\min }=C_{44}$ & $0.967 \pm 0.005$ & & 0.94 \\
\hline \multirow{2}{*}{ Poisson's ratio, $\nu$} & $\nu_{\max }=\nu\langle 110,1 \overline{1} 0\rangle$ & 0.54 & \multirow{2}{*}{$\cdots$} & 0.57 \\
\hline & $\nu_{\min }=\nu\langle 110,001\rangle$ & 0.33 & & 0.33 \\
\hline \multirow{2}{*}{ Anisotropy measure } & Zener, $A$ ( $=1$ if isotropic $)$ & 0.73 & $\cdots$ & 0.70 \\
\hline & $E_{\max } / E_{\min }$ & 1.35 & 1.22 & 1.42 \\
\hline
\end{tabular}


TABLE II. Isotropic aggregate properties of ZIF-8 based on the VRH averages, which are representative of a texture-free polycrystalline material. All properties are in GPa, apart from $\nu$, which is dimensionless.

\begin{tabular}{lllll}
\hline \hline & \multicolumn{4}{c}{ Isotropic elastic properties } \\
Method & \multicolumn{1}{c}{$E_{\mathrm{VRH}}$} & \multicolumn{1}{c}{$G_{\mathrm{VRH}}$} & \multicolumn{1}{c}{$K$} & $\nu_{\mathrm{VRH}}$ \\
\hline Brillouin scattering & $3.145 \pm 0.013$ & $1.095 \pm 0.005$ & $7.751 \pm 0.011$ & 0.43 \\
$\mathrm{~A} b$ initio B3LYP & 3.15 & 1.09 & 9.23 & 0.44 \\
\hline \hline
\end{tabular}

In comparison with conventional open-framework materials such as inorganic zeolites, the acoustic velocities and the corresponding $C_{i j}$ 's observed here are distinctly lower. Thus, Brillouin scatterings of inorganic zeolites (e.g., $[15,16]$ on rather denser crystals containing extraframework species) have all revealed transverse wave velocities that are a factor of 3 times greater $\left(T_{\mathrm{S}}\right.$ and $T_{\mathrm{F}}>3 \mathrm{~km} \mathrm{~s}^{-1}$ ) than in ZIF-8; the longitudinal wave velocities in zeolites are also significantly faster $(L>$ $6 \mathrm{~km} \mathrm{~s}^{-1}$ ). Furthermore, since the densities of inorganic zeolites are approximately twice those of ZIFs [3], the elastic constants of zeolites are about an order of magnitude higher than those of ZIF-8; their values typically fall in the range $20 \leq C_{i j} \leq 120 \mathrm{GPa}$ (see [9]). For example, the naturally occurring aluminosilicate mineral, zeolite chlorosodalite, has elastic constants from ultrasonic measurements [15] that are (in GPa) $C_{11}=88.52(71), C_{12}=$ $38.70(50)$, and $C_{44}=36.46(33)$, compared with $C_{11}=$ 9.522(7), $\quad C_{12}=6.865(14), \quad$ and $\quad C_{44}=0.967(4)$ for ZIF-8. Structural flexibility of inorganic zeolites is well documented; their elastic deformation is governed by the bending of $T$-O- $T$ angles connecting the rigid tetrahedra $(T)$ [17]. This comparison indicates that the characteristic $M$-Im- $M$ linkages in ZIFs are more compliant and can afford greater flexibility.

For crystals with cubic symmetry, the degree of elastic anisotropy can be straightforwardly quantified by the Zener ratio [18], $A=C_{44} /\left[\frac{1}{2}\left(C_{11}-C_{12}\right)\right]$, which represents the ratio of the two extreme shear coefficients (Fig. 2). We note that the numerator consists of the shear elastic constant $C_{44}$, which signifies resistance to shear on the $\{100\}$ planes when a pair of opposing shear forces are acting in the $\langle 0 k l\rangle$ directions [Fig. 2(b)]. In a similar fashion, the denominator $\frac{1}{2}\left(C_{11}-C_{12}\right)$ represents resistance to shear on the $\{110\}$ planes with forces acting along the $\langle 1 \overline{1} 0\rangle$ directions [Fig. 2(f)]. As such, the Zener ratio $A$ amounts to unity for a truly isotropic material when $C_{44}=\frac{1}{2}\left(C_{11}-C_{12}\right)$. Using the experimental $C_{i j}$ 's (Table I), we obtained $A=0.73$, which is in line with our $a b$ initio calculations that predict an $A$ value of 0.70 ; ZIF-8 is thus moderately anisotropic. Specifically, $A<1$ arises in ZIF- 8 because its minimum shear modulus is $G_{\min }=C_{44}=0.97 \mathrm{GPa}$ [Figs. 2(c) and 2(d)], whereas its maximum shear modulus is $G_{\max }=$ $\frac{1}{2}\left(C_{11}-C_{12}\right)=1.33 \mathrm{GPa}$ [Figs. 2(g) and 2(h)]. In the context of open-framework materials, the shear modulus
$G$ (ratio of shear stress $\tau$ to shear strain $\gamma$ ) is a measure of the framework rigidity [3] against structural distortion imposed by external shear forces. Here, the detailed anisotropic shear behavior of ZIF-8 is best understood

(a)

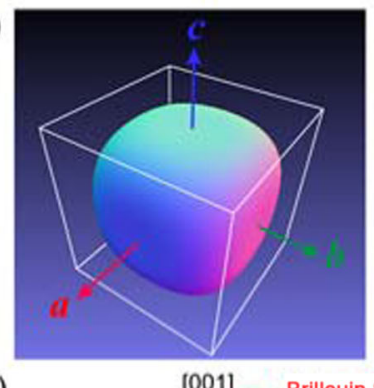

(c) $\quad \stackrel{[001]}{1} \ldots$ Brillouin Spect

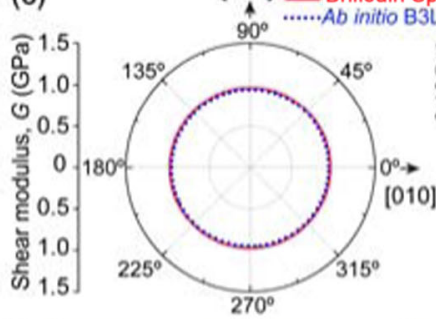

(e)
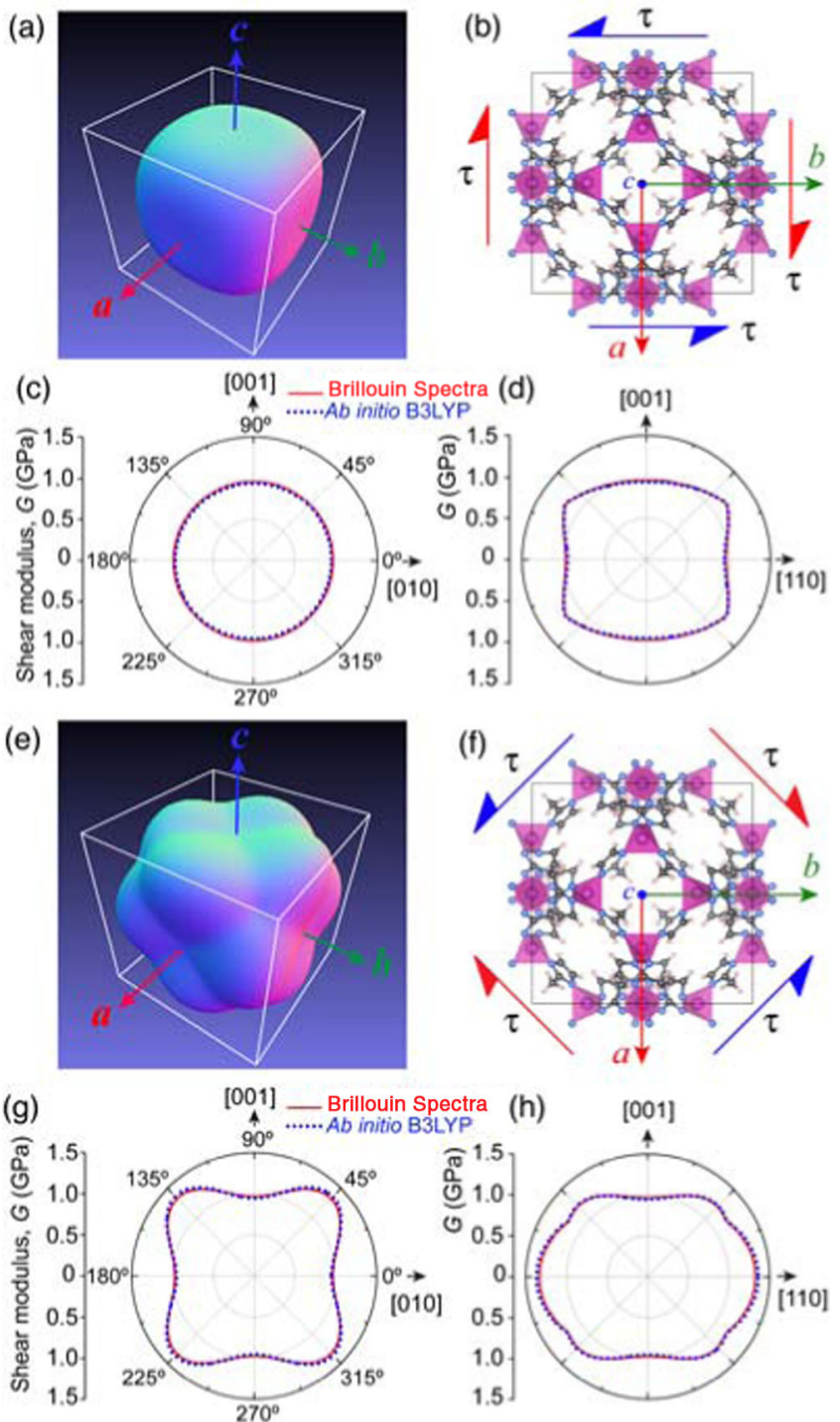

FIG. 2 (color online). Shear modulus $G$ representation surfaces. (a) 3D surface of $G_{\min }$ derived from Brillouin measurements. (b) Opposing shear stresses $\tau$ corresponding to $G_{\min }$. (c), (d) 2D polar plots of $G_{\min }$ from experiments and theory, projected onto the (100) and (110) planes, respectively. (e) $G_{\max }$ surface derived from experimental $C_{i j}$ 's. (f) Shear stresses that yield $G_{\max } \cdot(\mathrm{g})$,(h) 2D polar plots of $G_{\max }$. 
via $3 \mathrm{D}$ representation surfaces for both the minimum and the maximum shear moduli [Figs. 2(a) and 2(e)]. The SOD structure of ZIF-8 is most susceptible to distortion when shear stresses are applied onto the opposite four-membered rings, normal to the crystallographic axes; one possible scenario is depicted in Fig. 2(b).

Notably, the shear modulus $(G)$ of ZIF-8 is around 30 times smaller than that of its inorganic counterpart and, indeed, of other zeolites [9]. It is remarkable that a MOF with excellent thermal stability and permanent porosity (stable structure upon solvation-desolvation $[8,10]$ ) can have such a small shear modulus $\left(G_{\min } \lesssim 1 \mathrm{GPa}\right)$. Such a low value has never before been measured in an extended solid bound entirely by strong covalent and coordination bonds. Using the Voigt-Reuss-Hill (VRH) averaging (Table II), the aggregate value for the shear modulus of ZIF-8 amounts to $G_{\mathrm{VRH}} \sim 1.1 \mathrm{GPa}$. In contrast, the rigidity of inorganic zeolites against shearing is appreciably stronger with $25 \leq G_{\mathrm{VRH}} \leq 40 \mathrm{GPa}[15,16]$. Another fascinating comparison can be drawn against the renowned cubic MOF-5 material, $\mathrm{ZnO}_{4}$ (1,4-benzenedicarboxylate) [19], whose $C_{i j}$ 's have been extensively calculated $[3,5,6]$, but direct comparison with experiments has not been made. While MOF-5 has a larger porosity (SAV 80\% [9]), its minimum shear modulus has been computed to be relatively higher with $1.16 \leq G_{\min } \leq 3.6 \mathrm{GPa}$ [3]. In light of our findings, future experimental studies are warranted to establish whether ZIFs with other topologies (e.g., ZIF-20, -68 [10]) and MOFs encompassing flexible frameworks \{e.g., COFs (covalent organic frameworks), MILs (Matérial Institut Lavoisier) [1]\} will exhibit similarly low $G_{\min }$.

Interestingly, amongst the numerous single-crystalline materials measured to date [20], the exceptionally low $G$ of ZIF-8 discovered here is matched in magnitude only by the most weakly bound molecular solids (e.g., carbamazepine, $G_{\text {min }}=0.73 \mathrm{GPa}$ [21]). We note, however, that the mechanism responsible for each system's low rigidity is fundamentally different. In ZIF-8, it is linked to the intrinsic nature of the flexible 3D framework, but, in molecular crystals in which there is no 3D connectivity, it is attributed to weak van der Waals interactions. Moreover, our $a b$ initio calculations reveal that the shear deformation in ZIF-8 is dominated by cooperative bending of the N-Zn-N (tetrahedral) and Zn-mIm-Zn (bridging) bond angles [9], both of which are linked to the pliant $\mathrm{ZnN}_{4}$ tetrahedra [22] and accommodated by the surrounding porosity. This mechanism is fundamentally distinct from that in zeolites comprising rigid $\mathrm{SiO}_{4}$ tetrahedra [17].

We now focus on Young's modulus $(E)$ and Poisson's ratio $(\nu)$. Since we have established that ZIF-8 is elastically anisotropic, tensorial analysis $[14,23]$ of the $C_{i j}$ 's was necessary to elucidate the direction-dependent $E$ and $\nu$. We see that the representation surface for Young's modulus [Fig. 3(a)] exhibits protuberances along (a)
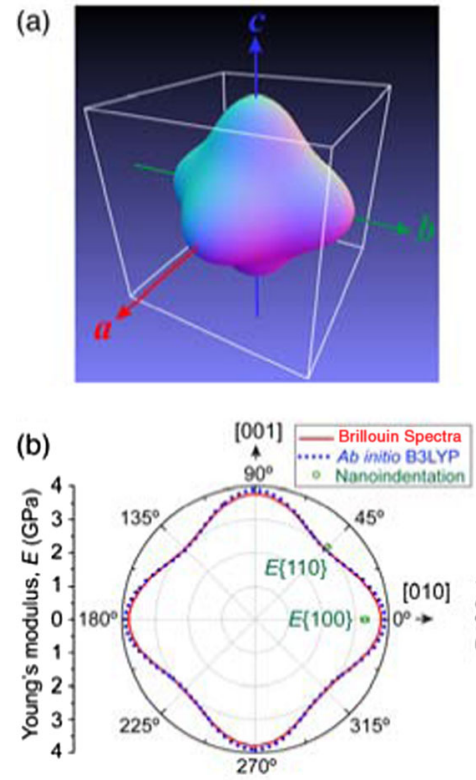

(e)

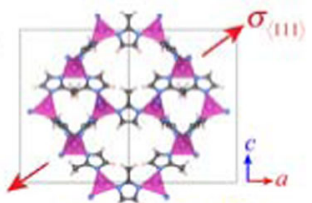

(d)
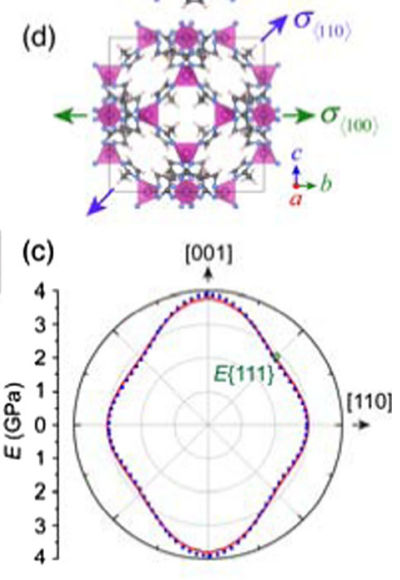

FIG. 3 (color online). Young's modulus $E$ representation surfaces. (a) 3D surface based on experimental $C_{i j}$ 's. (b),(c) Polar plots projected onto the (100) and (110) planes, respectively; the nanoindentation measurements are also plotted (Table I). (d), (e) Three sets of normal stresses $\sigma$ corresponding to the maximum, the intermediate, and the minimum stiffnesses.

the cube axes, such that $E$ is a maximum for the $\langle 100\rangle$ cube axes $\left[E_{\max }=3.77 \mathrm{GPa}\right.$ normal to the SOD four-membered ring; see Figs. 3(b) and 3(d)] and is a minimum along the $\langle 111\rangle$ body diagonals $\left[E_{\min }=\right.$ $2.78 \mathrm{GPa}$ normal to the SOD six-membered rings; see Figs. 3(c) and 3(e)]. It follows that Young's modulus anisotropy for ZIF-8, $E_{\max } / E_{\min }=1.35$, is appreciably lower than the value computed for MOF-5, which is therefore more anisotropic ( $E$ ratio 3.77 [5]). We note that all the important features of the experimental $E$ profile of ZIF-8 are correctly captured by our ab initio calculations [Figs. 3(b) and 3(c)]. Furthermore, our theoretical results reveal that the deformation mechanism during uniaxial straining involves stretching (or compression) of the $\mathrm{Zn}-\mathrm{N}$ bonds, coupled with bending of both the $\mathrm{N}-\mathrm{Zn}-\mathrm{N}$ and $\mathrm{Zn}-\mathrm{mIm}-\mathrm{Zn}$ bond angles, while the imidazolate rings remain rigid [9].

The representation surfaces for Poisson's ratio $(\nu)$ are shown in Fig. 4(a). We found that $0.33 \leq \nu \leq 0.54$, showing that $\nu$ remains positive (no negative Poisson's ratio); note that the range of $\nu$ is less extreme than in cubic crystals that are even more anisotropic [24]. Notably, the exceptionally low shear modulus of ZIF-8 is accompanied by a correspondingly high Poisson's ratio, which exceeds the typical maximum of 0.5 (in isotropic materials [4]). As shown in Figs. 4(b) and 4(c), Poisson's ratio is a maximum $\left(\nu_{\max }\right)$ when the framework structure is axially stretched along the $\langle 110\rangle$ face diagonal, leading to a lateral contraction along $\langle 1 \overline{1} 0\rangle$. Under the same loading condition, 


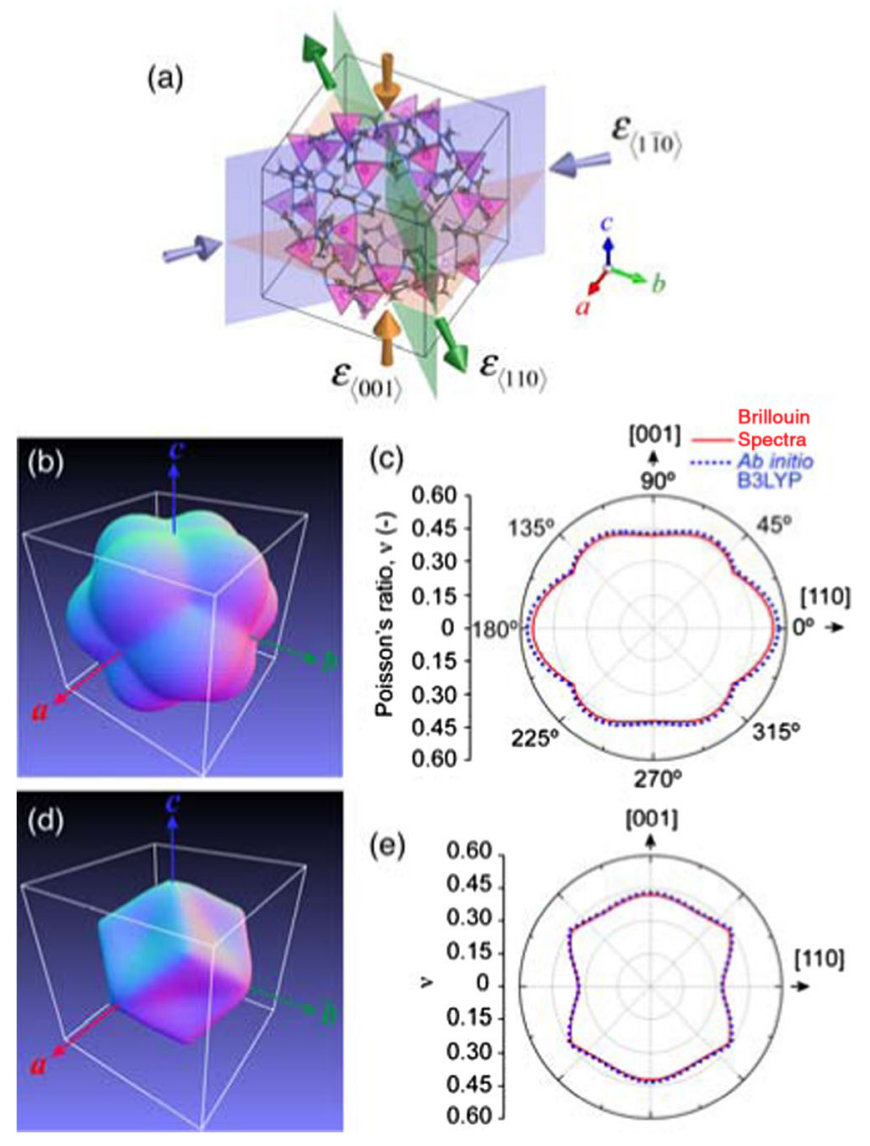

FIG. 4 (color online). Poisson's ratio $\nu$ representation surfaces. (a) Illustration depicting the axial and lateral directions in ZIF8's sodalite cage that yield $\nu_{\max }$ and $\nu_{\min }$. (b) 3D surface for $\nu_{\max }$ derived from measured $C_{i j}$ 's. (c) Polar plots of the measured and predicted $\nu_{\max }$ down the $[1 \overline{1} 0]$ axis. (d),(e) 3D surface and the corresponding polar plots for $\nu_{\min }$.

Poisson's ratio becomes a minimum $\left(\nu_{\min }\right)$ with respect to a lateral contraction along the $\langle 001\rangle$ cube axes [Figs. 4(d) and 4(e)]. Again, it is noteworthy that our ab initio calculations [Figs. 4(c) and 4(e)] can provide an accurate prediction of every complex variation observed in Poisson's ratio surface derived from Brillouin spectroscopy.

Another important elastic property is the bulk modulus $K$ (inverse of compressibility); it represents the resistance of the structure against volumetric strains under a hydrostatic pressure. Based on the adiabatic $C_{i j}$ 's from Brillouin scattering, we established that $K=7.751(11) \mathrm{GPa}$ at $295 \mathrm{~K}$, which is $\sim 19 \%$ greater than that determined by high-pressure crystallography $\{K=6.52(35) \mathrm{GPa}[25]\}$. Significantly, the bulk modulus of ZIF-8 is at least a factor of 7 times greater than its shear modulus; thus, ZIF-8's sodalite structure is considerably less compressible than it is rigid $(K \gg G)$. Finally, by comparison, ab initio calculations yield a yet higher $K=9.23 \mathrm{GPa}$ at $0 \mathrm{~K}$, signifying a marked reduction in compressibility when lattice vibrations become insignificant.
To put our findings into perspective, we note that technological materials rarely encounter uniform loadings during device fabrication and service. In practice, therefore, the complex stresses experienced by MOFs are more likely to be dominated by shear. On this basis, an exceptionally low shear modulus implies that there is a propensity for shear-induced plasticity, amorphization, and rupture. Our findings concerning the shearing characteristics of ZIF-8 are believed to be representative of other MOF-type materials featuring large porosity and flexible coordination polyhedra. The evidence is now quite compelling and raises questions about the viability of using flexible MOFs in certain commercial applications, which would expose them to significant shear distortions.

With the benefit of hindsight, we can explain several important physical phenomena reported for ZIF-8 that, hitherto, have not been fully understood. First, compared with hydrostatic compression (zero shear), the low shear modulus of the ZIF-8 framework explains why a nonhydrostatic compression triggers a more rapid loss of crystallinity [25]. Second, while ZIFs based upon substituted imidazolates, like ZIF-8, are resistant to temperatureinduced amorphization, they can be irreversibly amorphized via ball milling [26] because extensive shearing induces framework distortion and collapse. Third, we can now explain the surprising result that a liquid lubricant containing ZIF-8 additives demonstrates excellent antiwear properties [27]; given our findings, this seems reasonable because ZIF-8's low shear resistance reduces friction at interfaces, thus promoting better wear protection. In conclusion, while the elastic properties of the prototypical MOF, ZIF-8, are indeed complex, a detailed understanding of its elasticity opens up new possibilities for tuning the physical properties for emerging applications.

We gratefully acknowledge funding from the ERC (J.C.T., T.D.B., and A.K.C.) and the EPSRC, U.K. (R. G. and C.M.-D.). L. V. thanks the Department of Physics at Michigan Technological University for the provision of computational resources. J.C.T. thanks Dr. A. Marmier for the E1AM code.

*akc30@cam.ac.uk

[1] G. Férey, Chem. Soc. Rev. 37, 191 (2008).

[2] S. Kitagawa et al., Angew. Chem., Int. Ed. 43, 2334 (2004); A. C. McKinlay et al., ibid. 49, 6260 (2010).

[3] J. C. Tan and A. K. Cheetham, Chem. Soc. Rev. 40, 1059 (2011).

[4] G. N. Greaves et al., Nature Mater. 10, 823 (2011).

[5] D. F. Bahr et al., Phys. Rev. B 76, 184106 (2007).

[6] S. S. Han and W. A. Goddard, J. Phys. Chem. C 111, 15185 (2007).

[7] Y.Q. Tian et al., Chem. Eur. J. 13, 4146 (2007).

[8] K. S. Park et al., Proc. Natl. Acad. Sci. U.S.A. 103, 10186 (2006). 
[9] See Supplemental Material at http://link.aps.org/ supplemental/10.1103/PhysRevLett.108.095502 for experimental methods, computational approaches, and additional figures and data.

[10] J. C. Tan et al., Proc. Natl. Acad. Sci. U.S.A. 107, 9938 (2010).

[11] H. Ledbetter, Mater. Sci. Eng. A 442, 31 (2006).

[12] R. Dovesi et al., Z. Kristallogr. 220, 571 (2005).

[13] B. Civalleri et al., CrystEngComm 8, 364 (2006); W. F. Perger et al., Comput. Phys. Commun. 180, 1753 (2009).

[14] J.F. Nye, Physical Properties of Crystals (Clarendon, Oxford, 1985).

[15] Z. Li et al., Appl. Phys. Lett. 55, 1730 (1989).

[16] C. Sanchez-Valle et al., J. Appl. Phys. 98, 053508 (2005); C. Sanchez-Valle et al., J. Chem. Phys. 128, 184503 (2008).

[17] A. Sartbaeva et al., Nature Mater. 5, 962 (2006).

[18] C. Zener, Elasticity and Anelasticity of Metals (University of Chicago Press, Chicago, 1948).
[19] H. Li et al., Nature (London) 402, 276 (1999).

[20] A. G. Every and A. K. McCurdy, Low Frequency Properties of Dielectric Crystals, Group III: Crystal and Solid State Physics, Landolt-Bor̈nstein Numerical Data and Functional Relationships in Science and Technology, New Series Vol. 29, Subvolume A: Second and Higher Order Elastic Constants (Springer-Verlag, Berlin, 1992).

[21] H. Mohapatra and C. J. Eckhardt, J. Phys. Chem. B 112, 2293 (2008).

[22] T. D. Bennett et al., Chem. Eur. J. 16, 10684 (2010).

[23] A. Marmier et al., Comput. Phys. Commun. 181, 2102 (2010).

[24] Z. A. D. Lethbridge et al., Acta Mater. 58, 6444 (2010).

[25] K. W. Chapman et al., J. Am. Chem. Soc. 131, 17546 (2009).

[26] T. D. Bennett et al., J. Am. Chem. Soc. 133, 14546 (2011).

[27] Q. Shi et al., Angew. Chem., Int. Ed. 50, 672 (2011). 